\title{
HIDROPESÍA TORACOABDOMINAL NO INMUNE ASOCIADO A MÚLTIPLES ANOMALÍAS FETALES.
}

\author{
THORACOABDOMINAL NOT IMMUNE HYDROPS ASSOCIATED WITH \\ MULTIPLE FETAL ANOMALIES. \\ Nicol López Ovando ${ }^{1}$,Micaela López Ovando ${ }^{1}$, Dr. Samuel Rocha².
} 1 Facultad de Medicina
"Dr. Aurelio Melean",
Universidad Mayor de Sann
Simon, Cochabamba-Bolivia.
2 Médico cirujano, Especialista
en neonatología Pediátrica en el
Hospital Maternologico Infantil,
Cochabamba - Bolivia.

Nombre: Nicol López Ovando Telf. y celular: +59163985835 Correo electrónico: nklov_2002@hotmail.com

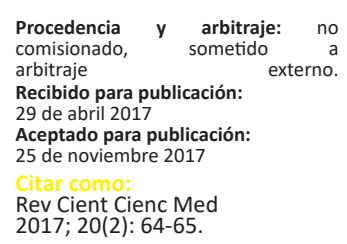

Recién nacido de sexo masculino, producto de 19,4 semanas obtenido por cesárea, peso de 1810 gr. Madre de 23 años, referida del Hospital de México de Sacaba al Hospital materno infantil German Urquidi Cochabamba- Bolivia, refiere cuadro clínico de aproximadamente 5 horas de evolución caracterizado por sangrado transvaginal , asociado a dolor abdominal tipo espasmódico, en hipogastrio que irradió a región lumbosacra y genitales externos, percibe movimientos fetales sin pérdida de líquido; antecedentes de Chagas y aborto hace 2 años, ,realizó 5 controles prenatales, porta ecografías donde se observa destrucción casi completa de hemisferios cerebrales, espacio supratentorial anecoico, manto cortical, cavidad torácica y abdominal unidas, aparente ausencia del diafragma, estructura llena de líquido, ascitis, pulmones hipoplásicos, corazón restringido, vísceras abdominales flotando, criptorquidia, ano imperforado dando en conclusión feto con

Figura 1. Recién nacido con hidropesía
$\begin{aligned} & \text { toracoabdominal } \\ & \text { craneofaciales. }\end{aligned}$

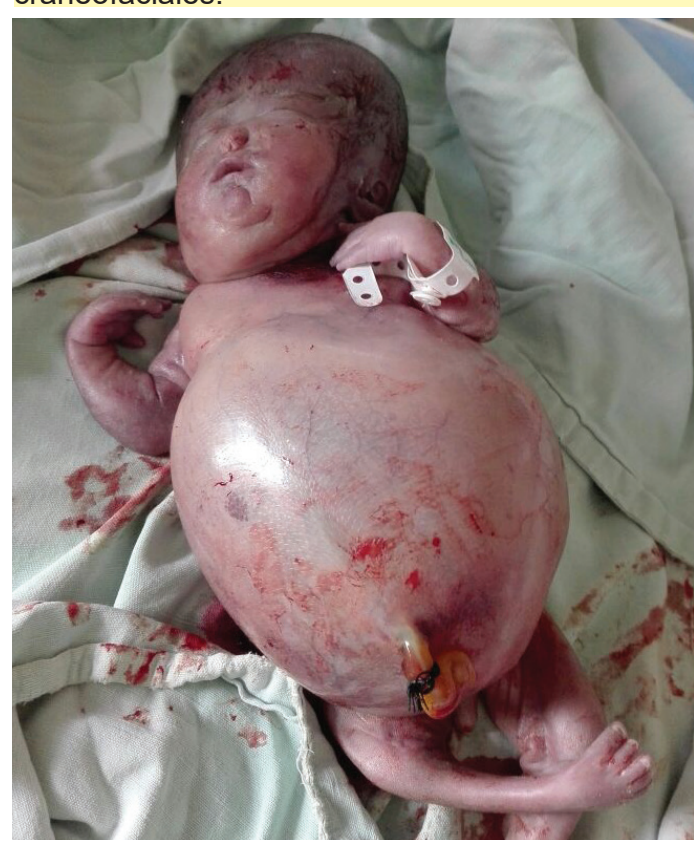

anomalías incompatibles con la vida, se procedió a una intervención y extracción del mismo.

Se llega al diagnóstico de hidropesía fetal no inmune (HFNI) asociado a diversas anomalías.

La hidropesía fetal es una condición en la cual se produce la acumulación anormal de líquido; agua corporal o intersticial ${ }^{1,2}$. Se divide en 2 tipos: inmune en $15 \%$ causado por el paso de anticuerpos maternos, contra los eritrocitos fetales, a través de la placenta y no inmune en $85 \%$ con ausencia de anticuerpos maternos ${ }^{3}$.

Las causas más frecuentes son: cardiovasculares (20\%), anomalías cromosómicas (15\%), trastornos hematológicos $(10 \%)$, infecciones (7-10\%), displasias linfáticas (5\%) síndromes monogénicos (4\%), metabolopatías (2\%) e idiopáticas $(20 \%)^{3,4}$. Se ha observado casos en las que la forma clínica de la enfermedad de Chagas se ha relacionado a esta condición ${ }^{5}$.

Figura 2. Recién nacido con criptorquídea, ano imperforado y genital externo mal desarrollado.

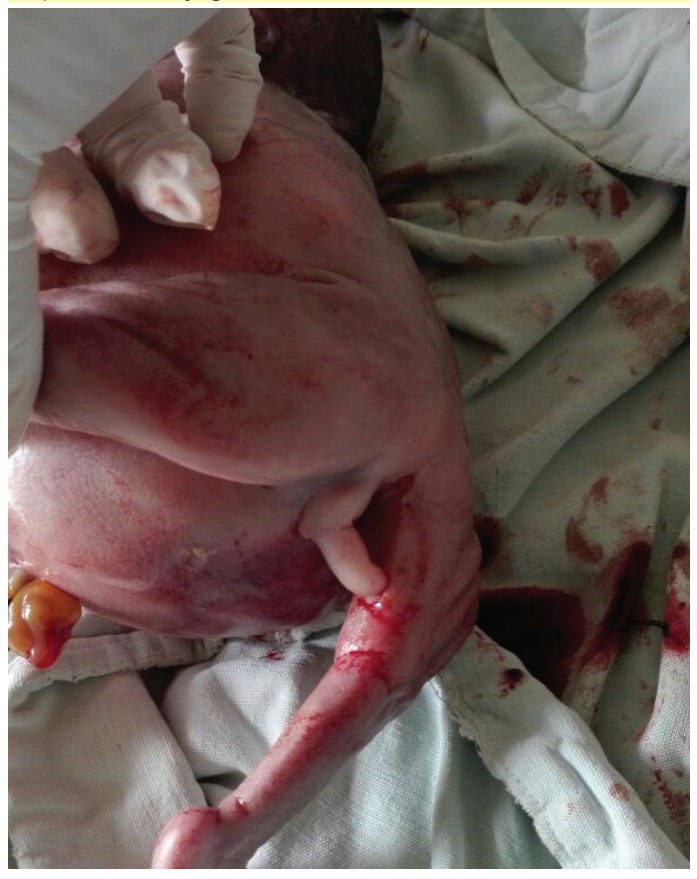

Rev Cient Cienc Méd Volumen 20, No $2: 2017$ 
El pronóstico del feto con HFNI en general es malo, la tasa de mortalidad depende del origen y magnitud, siendo este de 40 a $98 \%$. Las anomalías anatómicas están relacionadas a un peor pronóstico, constituyendo situaciones letales en casi todos los casos ${ }^{4}$.

Sólo los casos de infección por citomegalovirus y arritmias cardíacas sin anormalidades estructurales tienen una resolución espontánea, con pronóstico regularmente bueno ${ }^{5}$.

\section{REFERENCIAS}

1. Donovan CP, Alejandra RT, Sergio RR Hidropesía fetal. Diagnóstico ultrasonográfico en el primer nivel de atención. Rev Med Inst Mex Seguro Soc 2011; 49 (2): 205-208. . Acceso 19 de septiembre de 2017. Disponible en: www.medigraphic. com/pdfs/imss/im-2011/im112r.pdf

2. Rodríguez MM, Mengana FL, Urrutia CM. Hidropesía fetal en una gestante con enfermedad hipertensiva grave. Presentación de un caso. Revista Finlay 2013; 3(3). Acceso 20 de septiembre de 2017 Disponible en: http://www.revfinlay. sld.cu/index.php/finlay/article/view/193
3. Borobio V, Goncé A. Hidrops fetal no inmune. Guía Clínica: Clínic Barcelona hospital Univesitari 2013:1-7

4. Gonzalo I, Berzosa J, Díaz-Miguel V. Hydrops fetal no inmune. Clin Invest Ginecol Obstet 2008 ; 35 (3):109-12 Acceso 20 de septiembre de 2017. Disponible en: www.elsevier. es/...clinica-e-investigacion-ginecologia-obstetricia

5. Gratacos E, Gomez R, Nicolaides K, Romero R, Cabero L: Medicina fetal. En: editorial medica panamericana. 1ra Ed; 2009: 552 